M. A. Gris, secrétaire, donne lecture de la communication suivante, adressée à la Société par M. J.-B. Verlot :

BXTRAIT D'UNE LETTRE DE M. J.-B. VEIRLOOT A M. DE SCHENEFEL.D.

Grenoble, 30 mars 1864.

.... Un de mes amis m'a communiqué dernièrement un manuscrit de Villars, notre célèbre botaniste dauphinois ; c'est un mémoire écrit pour être adressé à l'Institut, et intitulé : Voyage de Grenoble à la Grande-Chartreuse, le 8 messidor an XII. J'ai fait une copie des parties de ce mémoire, les plus intéressantes au point de vue botanique (en négligeant tout ce qui concerne la minéralogie et la géologie), et je m'empresse, avec l'autorisation du propriétaire de l'autographe, de vous envoyer cette copie, espérant que, si vous voulez bien la communiquer à la Société botanique, la narration et les différents faits signalés par Villars intéresseront nos savants confrères.

Il n'est pas bien certain que ce travail de Villars ait été communiqué a l'Institut; car on se demande pourquoi, s'il l'avait été, l'Institut ne l'aurait pas gardé. On trouve seulement écrits en tête de la première page (par une main autre que celle de Villars)ces mots : "Arrivé le 10 thermidor an XIII." Est-ce là la date de l'arrivée du mémoire à Paris ? Je l'ignore. Mais, en compulsant les procès-verbaux de l'Institut de l'an XIII, on parviendrait peutêtre à savoir s'il a été réellement présenté à la savante compagnie.

Parmi les plantes citées dans ce mémoire, les plus intéressantes à mon avis sont :

$1^{\circ}$ Le Salix appendiculata Vill, qui, comme le pense M. Grenier $(\mathrm{Fl}$. de $F r$. III, p. 135), est identique avec le $S$. grandifolia Seringe. Le nom donné à la plante par Villars devra assurément, en raison de sa date (1789), être préféré à celui de Seringe.

$2^{\circ}$ Le Gentiana hybrida Vill., que Villars dit avoir trouvé dans les montagnes granitiques d'Uriage, Allevard, etc. Cette forme est mentionnée dans le Prodromus (t. IX, p. 87) par N. Grisebach, qui s'est aidé de ce que Villars en a communiqué et que Rœmer a publié, en 1809, dans son livre intitulé: Collectanea ad omnem rem botanicam spectantia. MLI. Grenier et Godron n'en parlent pas dans leur Flore de France.

$3^{\circ}$ Le Draba OEderi. La description qu'en donne Villars semble s'appliquer à la plante qu'on nomme aujourd'hui Draba nivalis DC. et que nous avons trouvée ensemble, en 1860, dans notre course au Lautaret et au Galibier. Mais quel est l'auteur de ce nom de Draba OEderi, que Villars n'indique que par l'initiale $\mathbf{F}$ et le $n^{\circ} 142$ ? Si l'on pouvait s'assurer du nom de cet auteur, il est évident que le nom de $\mathrm{Dr}$. OEderi devrait, par droit d'an- 
tériorité, être préféré à ceux de $\mathrm{Dr}$. nivalis Willd., Dr. nivalis DC. Syst. et Dr. Johannis Koch (1).

Quant aux citations des Rheum compactum, Rh. Rhaponticum et Polemonium caruleum, elles concernent des plantes échappées des cultures du couvent; il en est de mème du Dianthus barbatus signalé par Mutel dans sa Flore du Dauphiné (édit. 2). Aujourd'hui, on ne retrouve aucune trace de de ces espèces à l'état sauvage.

\section{VOYAGE DE GRENOBLE A LA GRANDE-CHARTREUSE, LE 8 MESSIDOR AN XII}

(27 JUIN 1804), par D. VIHLA RRS (2).

Arrivé le 10 thermidor an xuI (29 juillet 1805.)
Sumus plerique ut peregrina et admiremur et venereasur, et ea quas in nostro sunt conspectu negligamus.

ScHEUchz. Itin. alp, praf. 1.

Le même jour que les papiers publics annoncèrent à Grenoble la mort de l'intrépide et savant Humboldt (3) a Acapulco, était destiné pour un voyage à la Grande-Chartreuse. Depuis vingt-cinq ans que j'habite Grenoble, j'y en ai fait plus de dix, avec divers savants de diverses nations. Ce pays, intéressant par son voisinage, par son élévation, ses vastes forêts, ses sites et ses productions naturelles, offre toujours un nouvel intérêt.

La Chartreuse, rendue célèbre depuis $\mathbf{1 1 0 0}$ par son fondateur, par la solitude, par l'isolement et le silence qui y règnent, le fut encore par le nombre de savants et par les botanistes que ce désert attira, mais surtout par les plantes rares qu'il produit.

Les manuscrits de Richer de Belleval, que possède et qu'a fait imprimer en partie le professeur Gilibert (de Lyon), nous ont appris que Belleval, fondateur du Jardin de botanique de Montpellier sous Henri IV, en 1610 vint

(1) Note de M. de Schoenefeld. - Suivant le désir de M. Verlot, j'ai fait quelqnes recherches sur le nom de Draba OEderi, mais je n'ai pu le trouver dans aucun livre. Cependant l'abréviation de Villars, F. 142, me paraît signifier simplement figure 142. De plus, Villars nous dit que Willdenow (Sp. III, 427) a considéré cette plante comme synonyme du $\mathrm{Dr}$. nivalis. Or, dans le Flora danica, précisément à la planche 142, se trouve représenté un Draba stellata, cité par Willdenow (Sp. III, 427) comme synonyme de son Draba nivalis. Je suis done porté à croire que le nom de $\mathrm{Dr}$. OEderi a été créé par Villars lui-mème pour désigner le $\mathrm{Dr}$. stellata du Flora danica (recueil qui a été longtemps publié sous la direction d'OEder) et pour le distinguer du Draba stellata Jacq. et du Dr. stellata Wahlenb. Il est probable aussi que ce nom de Dr. OEderi n'existe nulle part ailleurs que dans le curieux autographe inédit que M. Verlot a bien vouln nous
mettre à même de publier. (2) Extrait d'un mémoire manuscrit de Villars, appartenant aujourd'hui (1864) à
M. Eugène Chaper (de Grenoble).

(3) L'annonce de la mort d'Alex. de Humboldt, à laquelle il est fait allusion ici, était heureusement une fausse nouvelle. L'illustre savant a encore vécu pendant cinquante-
cinq années depuis cétte époque, et n'est mort qu'en 1859 .

(Note de la Commission du Bulletin.) 


\section{$2 \mathrm{BHL}$ Biodiversity Heritage Library}

Verlot, J.-B. 1864. "Extrait D'une Lettre A M. De SchDnéfeld. 'Bulletin de la Société botanique de France 11, 151-152.

https://doi.org/10.1080/00378941.1864.10827331.

View This Item Online: https://www.biodiversitylibrary.org/item/8635

DOI: https://doi.org/10.1080/00378941.1864.10827331

Permalink: https://www.biodiversitylibrary.org/partpdf/159762

\section{Holding Institution}

Missouri Botanical Garden, Peter H. Raven Library

\section{Sponsored by}

Missouri Botanical Garden

\section{Copyright \& Reuse}

Copyright Status: Public domain. The BHL considers that this work is no longer under copyright protection.

This document was created from content at the Biodiversity Heritage Library, the world's largest open access digital library for biodiversity literature and archives. Visit BHL at https://www.biodiversitylibrary.org. 